\title{
THE SPECTRA OF EARLY-TYPE STARS IN THE NEAR ULTRA-VIOLET REGION
}

\author{
P. SWINGS ${ }^{\mathrm{I}}$ and M. DÉSIRANT
}

\section{ABSTRACT}

The spectra of three B stars and of eleven A stars have been measured for wavelengths in the region $3570-3930 \mathrm{~A}$. Identifications are made for most of the lines, and a discussion of the behavior of the elements is given.

The spectrograms used in this investigation were obtained with the one-prism Yerkes spectrograph, which was attached to the 69inch reflector of the Perkins Observatory. Most of the plates could be measured as far into the violet as $\lambda 3680$; one plate of $a$ Cygni was measured as far as $3570 \mathrm{~A}$. The scale of the spectrograms is $15.4 \mathrm{~A}$ per millimeter at $\lambda 3780$. For the reduction of the measures quadratic formulae were used.

The stars investigated, together with their Harvard spectral types, are given in Table I.

$\gamma$ Pegasi $\mathrm{B}_{2}$
$\gamma$ Orionis $\mathrm{B}_{2}$
$\beta$ Orionis $\mathrm{B} 8 \mathrm{p}$
$\alpha$ Lyrae Ao
$\gamma$ Geminorum Ao
$a$ Cygni A2p
$a$ Andromedae Aop

\section{TABLE I}

$\theta$ Aurigae Aop
$a$ Canum Venaticorum Aop
$\epsilon$ Ursae Majoris Aop
$\theta$ Leonis Ao
$a$ Geminorum Ao
$a$ Canis Majoris Ao
$\gamma$ Lyrae Aop

For the identifications Miss Moore's table ${ }^{2}$ was found to be invaluable; Kaiser's tables and the Revised Rowland were also used. ${ }^{3}$

In the case of the B stars, a number of lines are added to Marshall' $s^{4}$ and to Struve's $s^{5}$ lists and the evidence for the identification of $\mathrm{P}^{++}$and $\mathrm{Al}^{+}$is strengthened. About 60,per cent of the lines may be considered as fairly well identified.

The only extended investigation of stellar spectra of type A in the

I Advanced fellow of the C.R.B. Educational Foundation.

${ }^{2}$ A Multiplet Table of Astrophysical Interest (Princeton, I933).

${ }^{3}$ For $\mathrm{Fe}^{+}$the new measures by Dingle have been used (M.N., 95, 704, 1935).

${ }_{4}$ R. K. Marshall, Pub. Obs. U. of Michigan, 5, No. I2, I934.

5 O. Struve, Ap. J., 74, 225, I93r. 
near ultra-violet region is that of $a$ Cygni by Wright. ${ }^{6}$ Table III includes in the common wave-length region many lines additional to those measured by him.

The results are collected in Tables II and III, which deal, respectively, with the B and A stars. In the last column of Tables II and III the figures in parentheses are the Rowland intensities in the solar spectrum; the figures in brackets are laboratory intensities. The identifications in braces are doubtful.

In Table III the second column contains the mean wave-lengths and intensities for the following stars: $a$ Lyr, $\gamma$ Gem, $\theta$ Leo, $a$ Gem, $a \mathrm{CMa}$, and $\gamma$ Lyr. These six spectra are very similar and have not been listed separately. The identifications listed in the last column of Table III cannot be considered as complete for every case; many of them are satisfactory, but for a number it is certain that other contributors will have to be considered. The identifications are probably about as complete as they can be made at this time.

The behavior of the elements in the different spectra agrees quite well, for the normal stars, with Morgan's description. ${ }^{7}$

\section{EXAMINATION OF THE INDIVIDUAL ELEMENTS ${ }^{8}$}

$H$. The Balmer lines are usually visible as far as $H \omega\left(=H_{26}=\right.$ $3667.87 \mathrm{~A}$ ).

$H e$. The strongest line in this region is $38 \mathrm{Ig} .6 \mathrm{I}$ [4], it is either missing in A spectra or blended with the red part of $3820.6 \mathrm{I}$. The fainter lines 3926.53 [I], 3867.54 [2+I], and 3838.09 [I], which coincide with observed absorption lines, are presumably chance coincidences.

$\mathrm{C}^{+}$. The strongest lines in this region are 3918.98 [6] and 3920.68 [8]; they probably contribute very little to any A-type spectrum.

$\mathrm{O}^{+}$. The two lines 39r9.28 [6] and 39Ir.95 [10], which are fairly strong in the B stars, are merely chance coincidences in the A stars; the other four lines, 3875.82 [4], 3864.45 [5], 3830.45 [4], and 3727.33 [8], are also chance coincidences, as they do not appear even in $\beta$ Orionis (B8).

${ }^{6}$ W. H. Wright, Lick Obs. Bull., No. 332, I921.

7 W. W. Morgan, Pub. Yerkes Obs., 7, Part 3, I935.

${ }^{8}$ The elements indicated here are only those for which the spectral region is favorable. 
TABLE II

\begin{tabular}{|c|c|c|c|c|}
\hline $\begin{array}{l}\text { Approximate } \\
\text { Wave-Length }\end{array}$ & $\begin{array}{c}\gamma \mathrm{Peg} \\
\mathrm{B}_{2}\end{array}$ & $\begin{array}{c}\gamma \text { Ori } \\
\mathrm{B}_{2}\end{array}$ & $\begin{array}{c}\beta \text { Ori } \\
\text { B } 8 p\end{array}$ & Identifications \\
\hline 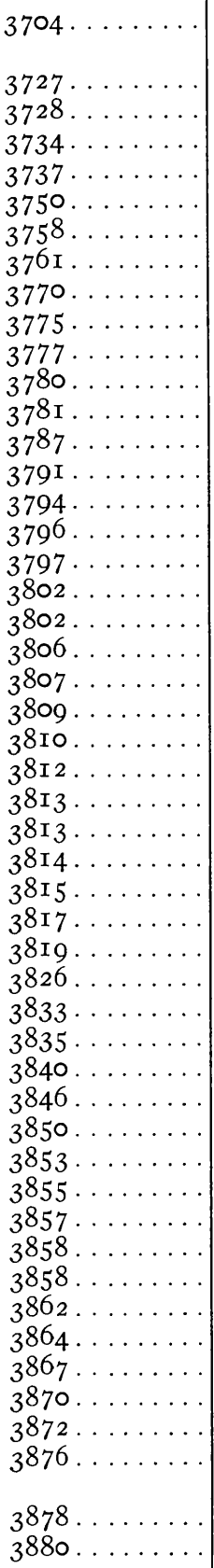 & 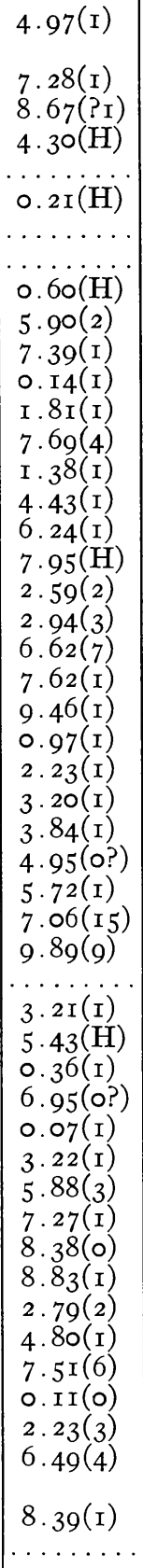 & 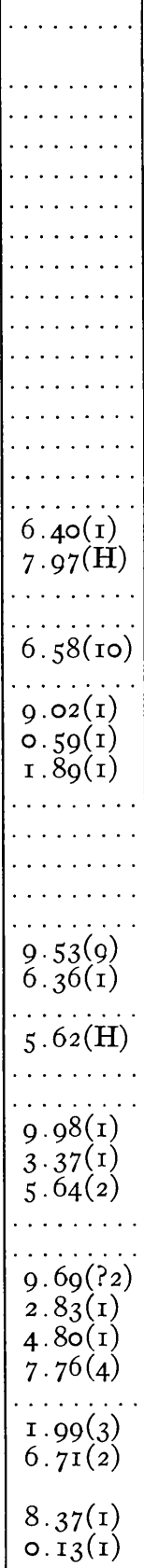 & 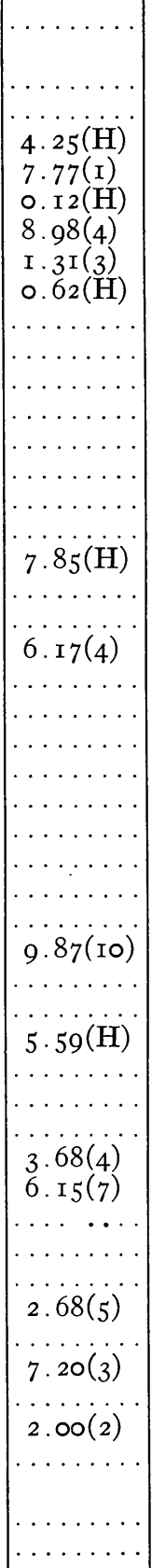 & 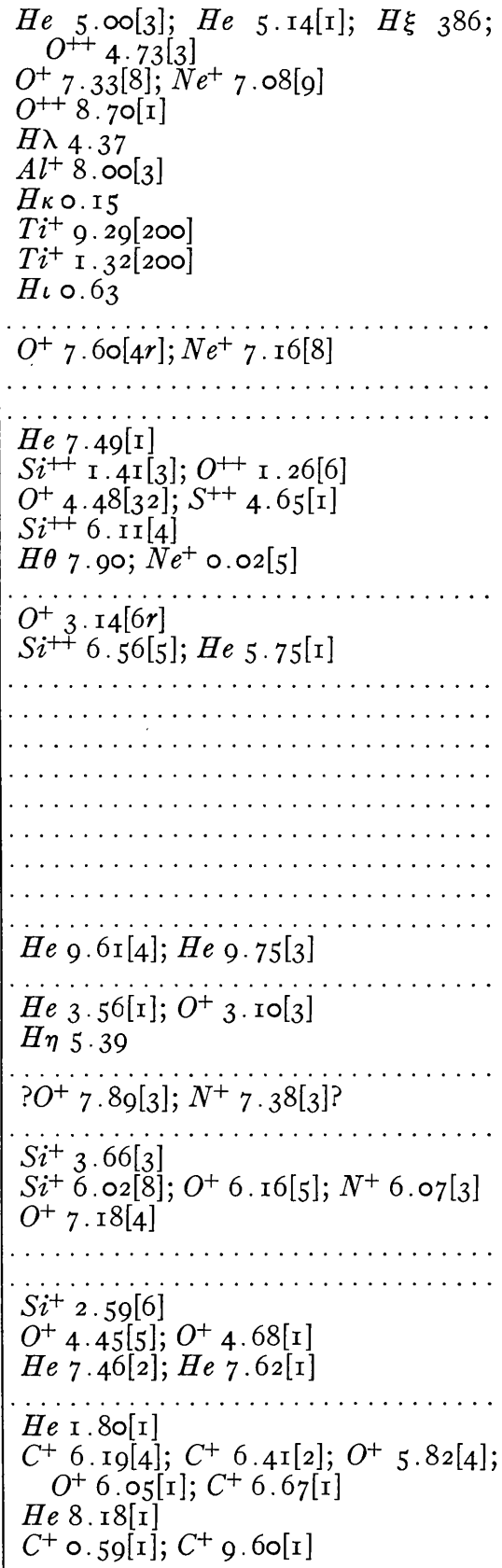 \\
\hline
\end{tabular}


TABLE II-Continued

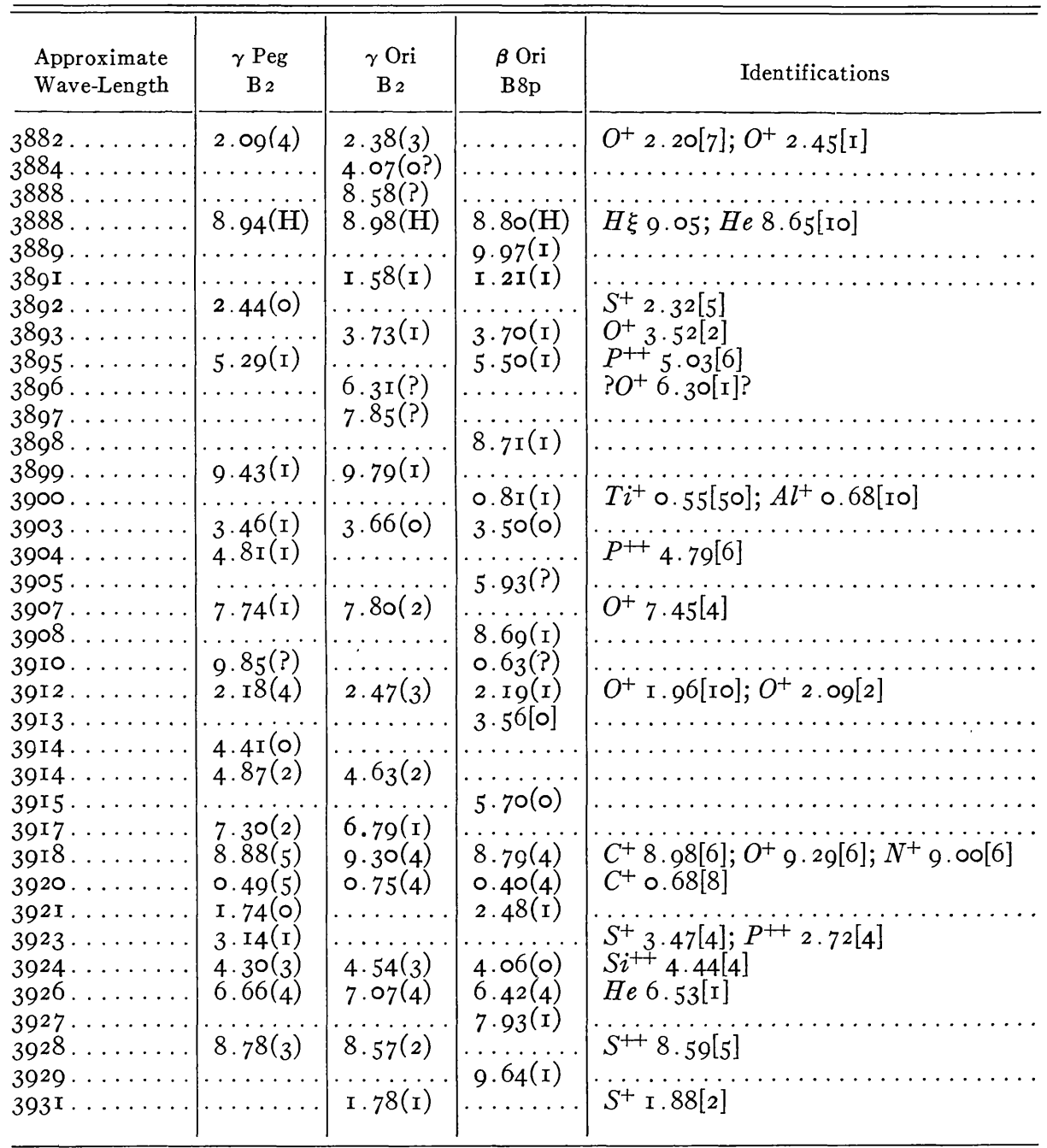

$N e^{+}$. The absence of 3777.I6 [8], which would be in a favorable region, seems to be strong evidence against the identification of $\mathrm{Ne}^{+}$; this agrees with the expected maximum intensity in the B stars. All the other coincidences are probably due to chance.

$M g$. The triplet 3838.30 [Ioor], 3832.3I [80r], 3829.37 [40] is very strong in all the A stars except in $a$ And and $\theta$ Aur, where the three lines are missing. The triplet is especially strong in a Lyr, $a \mathrm{Gem}, \epsilon \mathrm{UMa}, \gamma \mathrm{Gem}$, and $a \mathrm{CMa}$. Usually the observed estimated 


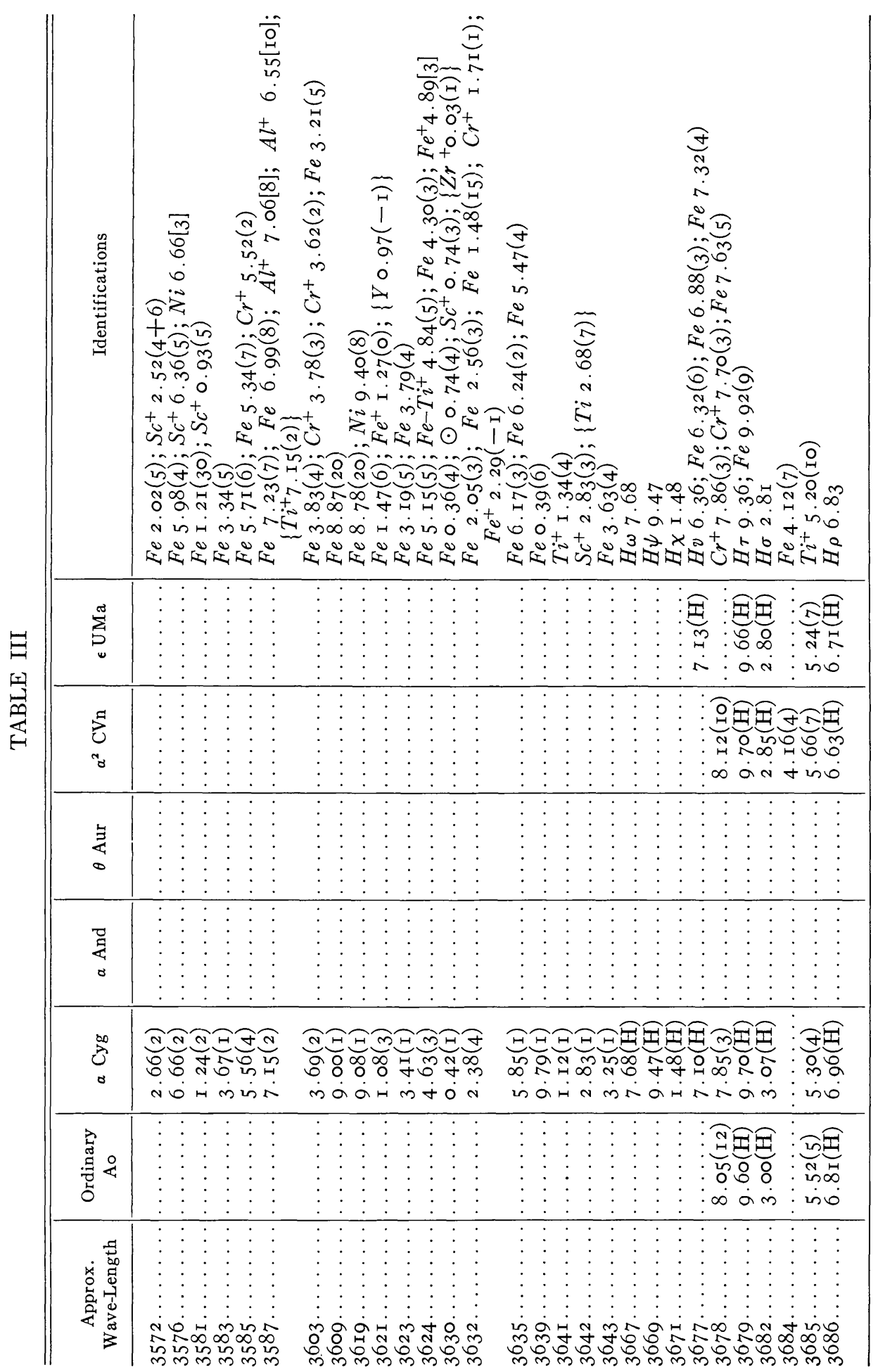




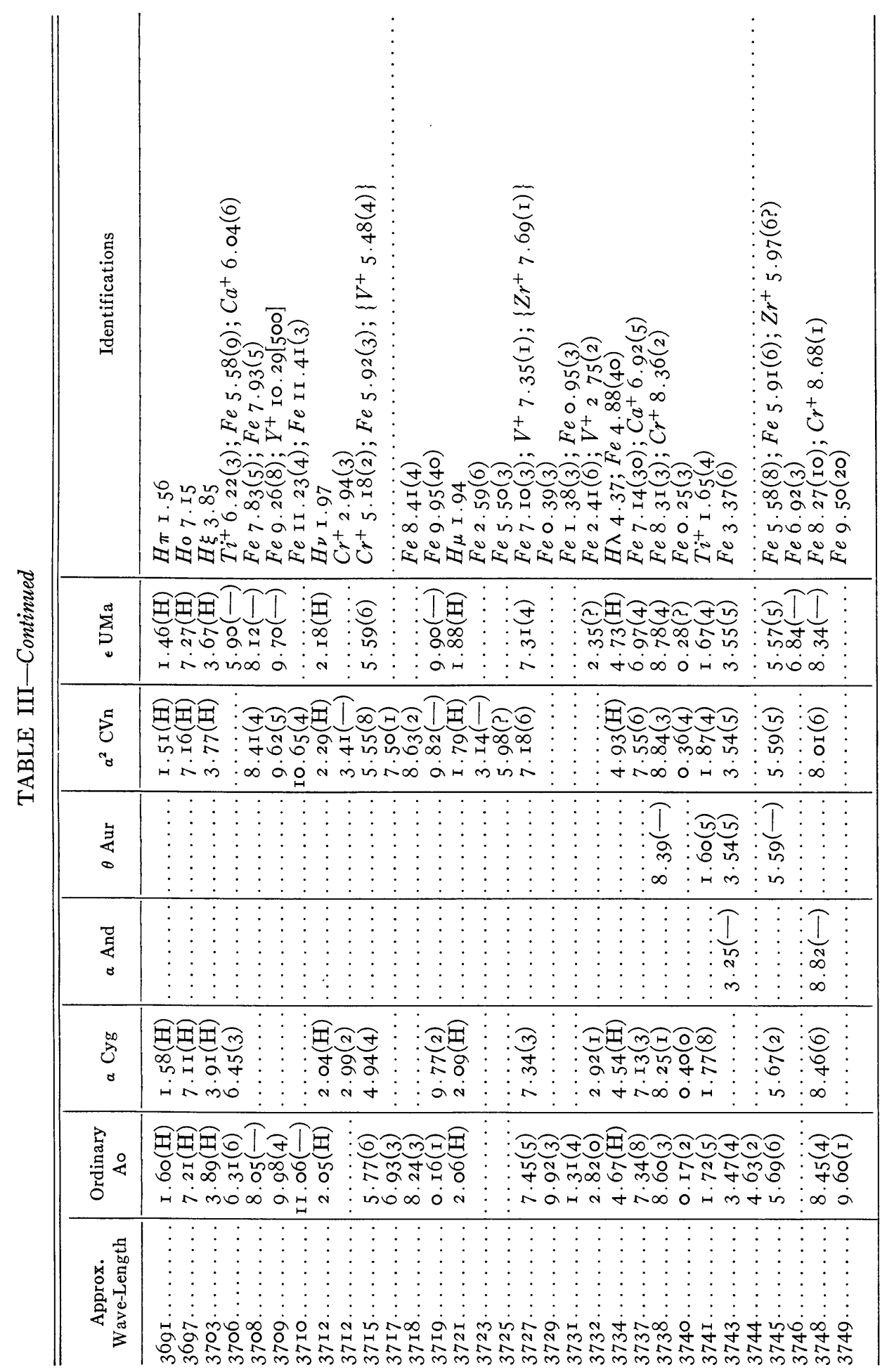




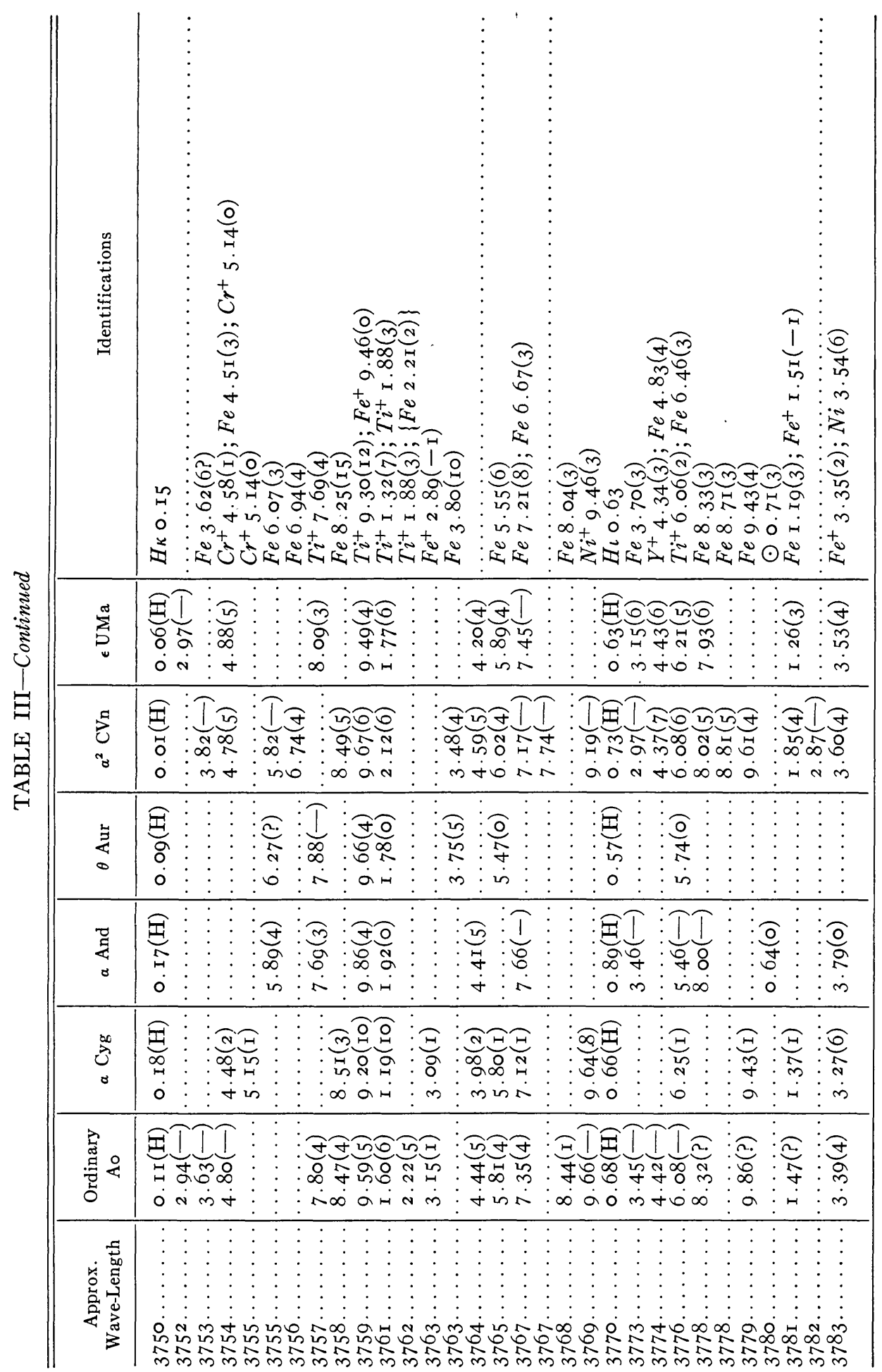




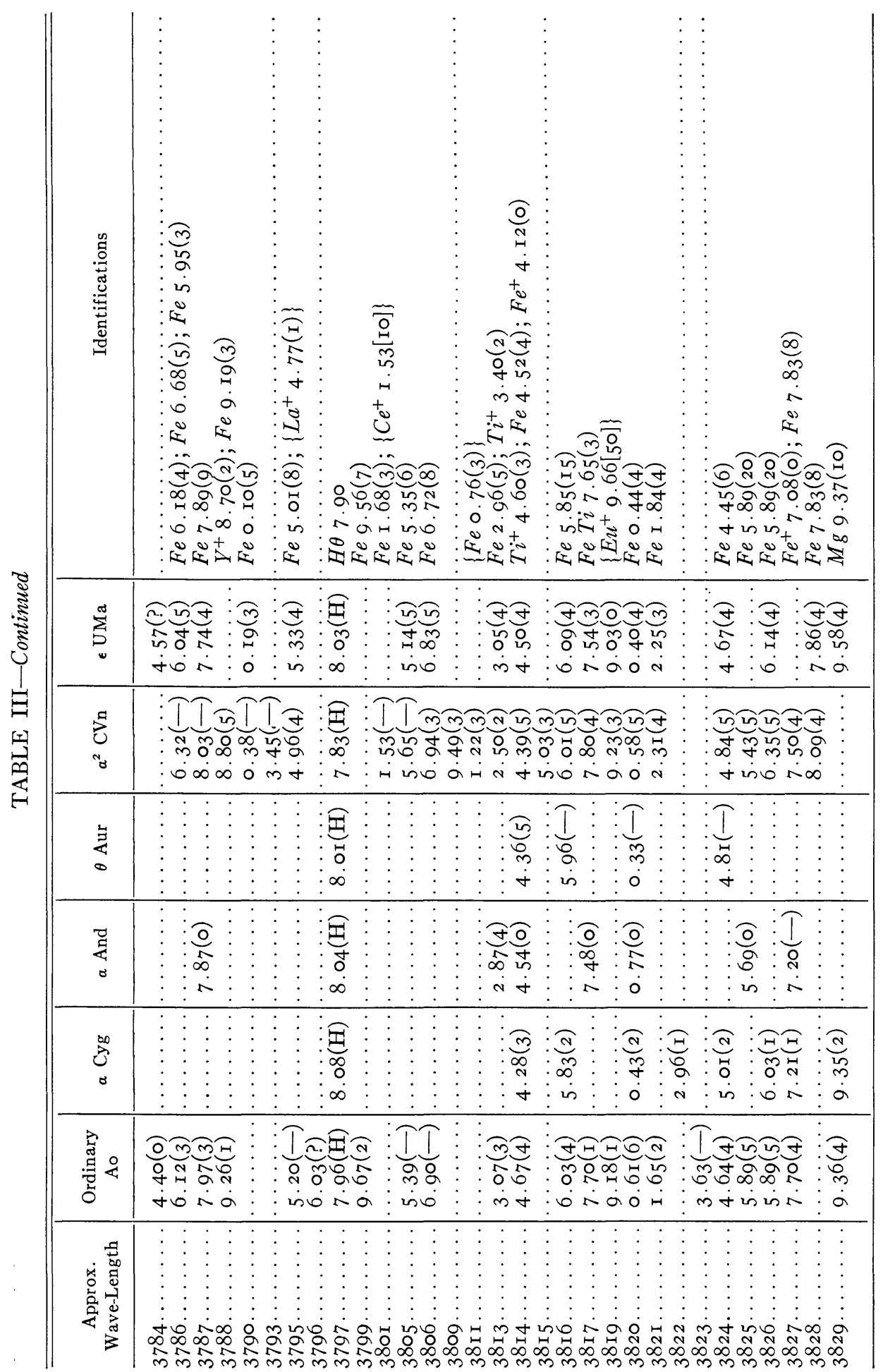




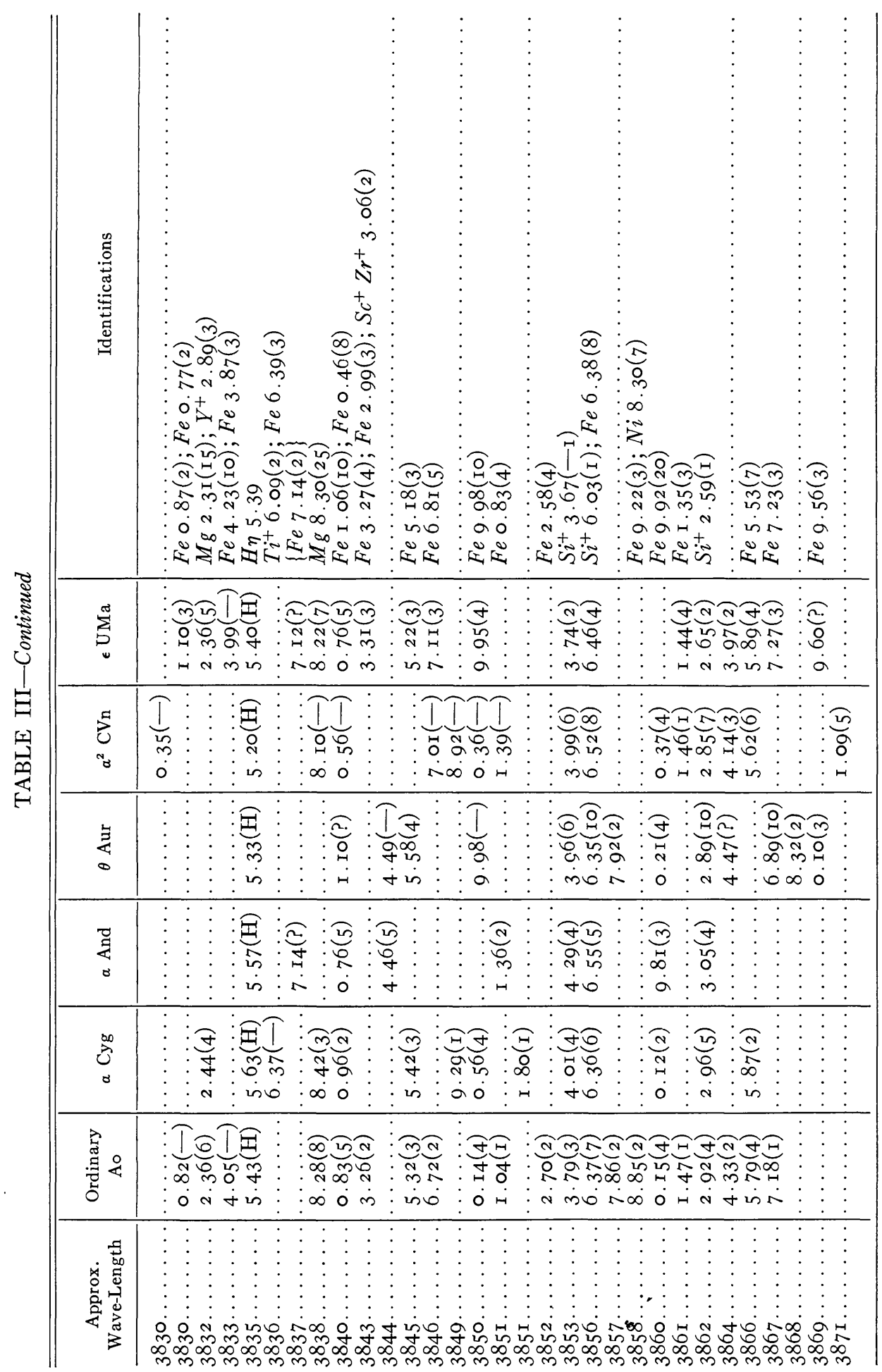




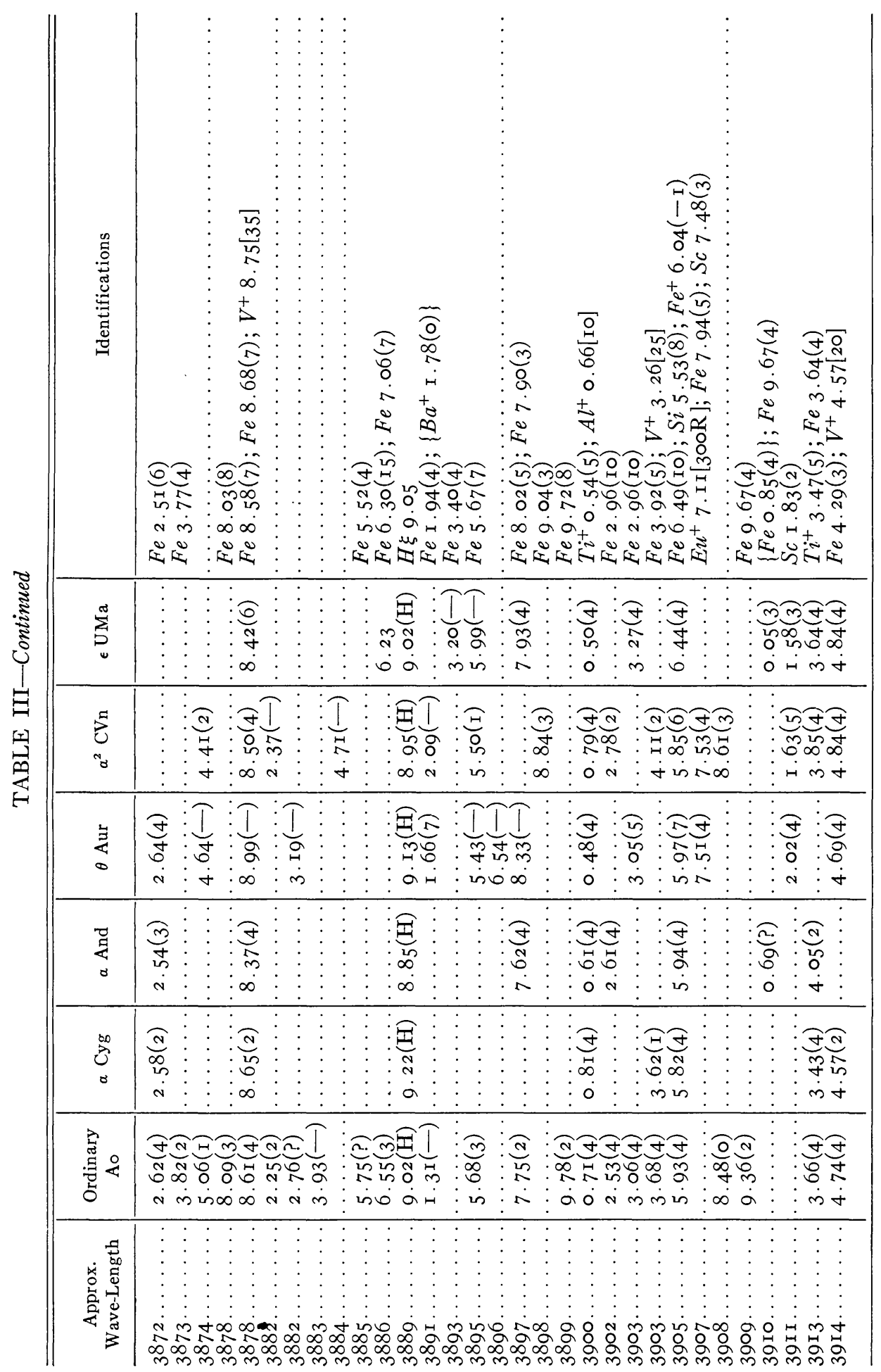




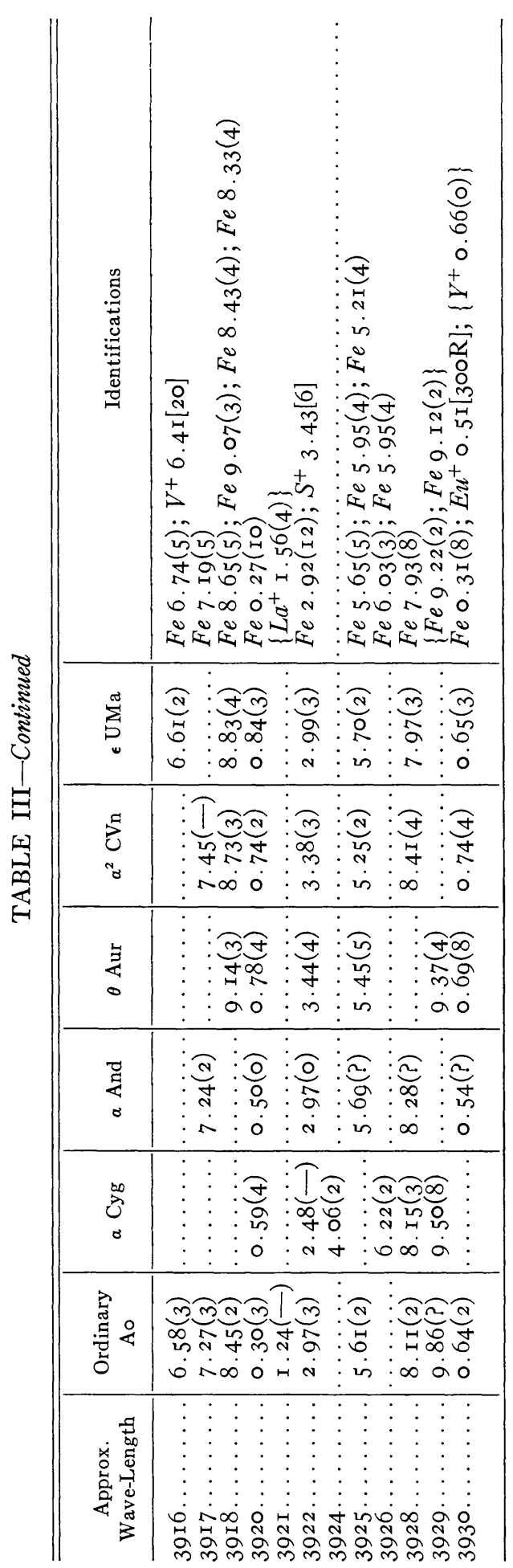


intensities agree quite well with the laboratory intensities, as is shown in Table IV. There are no strong singlet lines in this region.

$\mathrm{Mg}^{+}$. The only lines in the region covered are 3848.24 [7] and $3850.40[6], 3^{2} \mathrm{D}-5^{2} \mathrm{P}^{\circ}$; as 3848.24 does not appear, the wave-length coincidence of 3850.40 seems due to chance.

$\mathrm{Al}^{+}$. The strongest line at 3900.66 [Io], is blended with $\mathrm{Ti}^{+}$ 3900.54 (5). The blend appears in all the stars except $\theta$ Leo and a CMa. The triplet 3586.55 [9], 87.06 [8], and 87.44 [7] may possibly be blended with $\mathrm{Fe} 3587.63$ (7), which is present in a Cyg.

$\mathrm{Si}$. The only strong line in this region is 3905.53 (8), which possibly blends with the $\mathrm{Fe}$ line 3906.49 (IO). Actually the mean stellar

TABLE IV

\begin{tabular}{|c|c|c|c|c|c|c|}
\hline & \multicolumn{6}{|c|}{ Magnesium Triplet $3^{3} \mathrm{P}^{\circ}-3^{3} \mathrm{D}$} \\
\hline & Lab. & $\odot$ & $a$ Lyr & $\epsilon \dot{\mathrm{U} M a}$ & $a \mathrm{Gem}$ & $a \mathrm{CMa}$ \\
\hline $\begin{array}{l}3838.30 \ldots \ldots \ldots \\
3832.31 \ldots \ldots \ldots \\
3829.37 \ldots \ldots \ldots\end{array}$ & $\begin{array}{r}100 \\
80 \\
40\end{array}$ & $\begin{array}{l}25 \\
\text { I } 5 \\
\text { IO }\end{array}$ & $\begin{array}{l}7 \\
4 \\
3\end{array}$ & $\begin{array}{l}7 \\
5 \\
4\end{array}$ & $\begin{array}{r}\text { I0 } \\
7 \\
6\end{array}$ & $\begin{array}{l}7 \\
5 \\
3\end{array}$ \\
\hline
\end{tabular}

wave-length is 3905.93; Wright's value in a Cyg is 3906.I. Both measures are definitely smaller than the $F e$ wave-length, indicating the possible influence of the $S i$ line. On the other hand, the observed line at 3905.93 is much stronger than 3920.50 , which is due to $\mathrm{Fe} 3920.27$ of the same intensity (IO) in the sun. A weak $\mathrm{Fe}^{+}$line at 3906.04 ( - I) may also contribute to the blend and complicate the question.

$\mathrm{Si}^{+}$. The three strong lines in the region are 3853.67 [3], 3856.03 [8], and 3862.59 [6]. These lines require a considerably lower excitation potential ( 6.83 volts) than the pair at 4I $28-4$ I 30 (9.80 volts). All three of the lines are present in all the stars except $\gamma$ Lyr. The intensities usually agree with the laboratory values, as is shown in Table V. There are no other $\mathrm{Si}^{+}$lines in this region.

$S^{+}$. There is no evidence for or against the presence of $S^{+}$in this region, as the strongest line, 3923.43 [6] is blended with $F e$ 3922.92.

$C a$. There seem to be no $C a$ lines in this region, as the only coincidence is 3624.63 in a Cyg, intensity 3 , which might be due to $C a$ 
3624.I 2 [20]; other members of the same multiplet, however, do not appear.

$\mathrm{Ca}^{+}$. The only lines in the region are 3706.04 [Io] and 3736.92 [I I]; they are blended with strong $\mathrm{Fe}$ lines, 3705.58 and 3737.I4.

$S c$. There are probably no stellar $S c$ lines in this region. A strong line at 39 r .83 [roo] coincides with an observed line in $\theta$ Aur, $a$ CVn,

TABLE V

$S i^{+}$LINES

\begin{tabular}{r|r|r|r|r|r|r|r|r|r|r|r|r}
\hline \hline & Lab. & $\beta$ Ori & $\alpha$ Lyr & $\gamma$ Gem & $\alpha$ Cyg & $\alpha$ And & $\theta$ Aur & $\alpha$ CVn & $\epsilon$ UMa & $\theta$ Leo & $\alpha$ Gem & $a$ CMa \\
\hline $3862.59 \ldots$ & 6 & 5 & 7 & 6 & 5 & 4 & Io & 7 & 2 & 4 & 4 & 3 \\
$3856.03 \ldots$ & 8 & 7 & 7 & 6 & 6 & 5 & I0 & 8 & 4 & I0 & 5 & 5 \\
$3853.67 \ldots$ & 3 & 4 & $3-4$ & 3 & 4 & 4 & 6 & 6 & 2 & 4 & 3 & 2 \\
\hline
\end{tabular}

TABLE VI

\begin{tabular}{|c|c|c|c|}
\hline $\operatorname{Lab} \lambda$ & Lab. Int. & $\begin{array}{l}\text { Int. in } \\
a \text { Cyg }\end{array}$ & Blends \\
\hline $\left.\begin{array}{l}3572.48 \\
3572.57\end{array}\right\} \ldots$ & 50 & 2 & $\mathrm{Fe}(5)$ \\
\hline $\left.\begin{array}{l}3576.33 \\
3576.39\end{array}\right\}$ & 30 & 2 & $\mathrm{Fe}(4)$ and $N i[3]$ \\
\hline $3580.93 \ldots \ldots$ & 20 & 2 & $\mathrm{Fe}(30)$ \\
\hline $3630.74 \ldots$ & I00 & I & $F e(3)$ \\
\hline 3642.83. & 50 & I & \\
\hline
\end{tabular}

and $\epsilon \mathrm{UMa}$; but the other line of the same intensity at 4023.69 was not observed by Morgan in $\theta$ Aur and $a \mathrm{CVn}$.

$S c^{+}$. The following lines, which are the strongest in the observed region, may be partly due to $S c^{+}$in the spectrum of $a$ Cyg. An examination of the observed intensities shows that $S c^{+}$must be a minor contributor. Morgan found rather weak lines of $\mathrm{Sc}^{+}$in the ordinary photographic region of $a$ Cyg (see Table VI).

$T i$. Many coincidences in wave-length occur, but they seem due to chance.

$\mathrm{Ti}^{+}$. This spectral region is very suitable for investigations of $\mathrm{Ti}^{+}$ because of the presence of very strong lines, such as 3759.30 [200], 
$376 \mathrm{I} .32$ [200], and 3685.20 [250]. The lines are strongest in $a$ Cyg; they are also very strong in $a \mathrm{CVn}, \theta$ Leo, and $a$ Gem.

$V$. The lines shown in Table VII may possibly contribute to blends, but their contribution is still doubtful.

$V^{+}$. The strongest lines-3878.75 [35], 3903.26 [25], and 3727.35 [35]-seem present, but they are blended with $F e$ lines, which leaves the identification of $V^{+}$doubtful in this region.

$\mathrm{Cr}$. Many coincidences occur, but they seem due to chance; there are no very strong $\mathrm{Cr}$ lines in this region.

$\mathrm{Cr}^{+}$. All lines of intensity $\geq 2$ in the solar spectrum are present in a Cyg; even several lines of intensity I in the sun may be present,

TABLE VII

\begin{tabular}{c|c|c|l}
\hline \hline Lab. $\lambda$ & Stellar $\lambda$ & $\begin{array}{c}\text { Mean } \\
\text { Observed } \\
\text { Intensity }\end{array}$ & \multicolumn{1}{|c}{ Blends } \\
\hline $3855.85[\mathrm{I00}] \ldots \ldots$ & 56.37 & 5 & $\mathrm{Si} 6.03 ; \mathrm{Fe} 6.38(8)$ \\
$3850.76[60] \ldots \ldots$ & 40.82 & 5 & $F e \mathrm{Mn}(\mathrm{I0}) ; \mathrm{Fe}(8)$ \\
$3828.57[60] \ldots \ldots$ & 28.10 & 4 & $F e(8)$ \\
$3818.24[60] \ldots \ldots$ & I 7.70 & 3 & $F e(3)$ \\
$3813.49[60] \ldots \ldots$ & I3.07 & 4 & $F e(5) ; \mathrm{T}^{+}(2)$ \\
$3703.59[100] \ldots \ldots$ & 04. I I & $\mathrm{H}$ & $\mathrm{H} \xi$ \\
\hline
\end{tabular}

but they are blended with $F e$. The lines are especially strong in a Cyg and also in $a \mathrm{CVn}$.

$M n$. The wave-length coincidences are due to chance; the spectral region does not contain strong $M n$ lines.

$M n^{+}$. The region is also unsuitable for the detection of $M n^{+}$. The peculiar stars, $a$ And and $a \mathrm{CVn}$, which show $\mathrm{Mn}^{+}$lines in the ordinary photographic region, contain no trace of 3883.28 , which is the strongest line of $M n^{+}$in this region.

$F e$. Very many lines are present; the region is particularly rich in lines of great laboratory intensity. The $F e$ lines are especially strong in $\theta$ Leo, $a$ CVn, $a$ CMa, $a$ Lyr, and $a$ Cyg.

$\mathrm{Fe}^{+}$. Rather few $\mathrm{Fe}^{+}$lines are in the region covered; they are strong in a Cyg.

Co. This element seems absent; the strong line 3845.57 [60] coincides with a stellar line; but 3894 . Io [60] of the same multiplet is absent; $\lambda 3_{3873.13}^{[60]}$ is also absent. 
$N i$. The region is very suitable for the detection of $N i$. Line 36 I9.40 [i5० R] may contribute to the stellar line 36r9.08 in a Cyg, which is mainly due to $\mathrm{Fe}{ }_{3} 618.78 ; \mathrm{Ni}_{3} 858.30$ [40r] may contribute to 3858.85 , which is principally due to $F e 9.22$. Other coincidences seem due to chance.

$\mathrm{Ni}^{+}$. The region is particularly suitable for the investigation of $\mathrm{Ni}^{+}$. The line 3769.46 [5] is strong in a Cyg, intensity 8; it also appears in $a$ CVn and $\theta$ Leo. The other strong line is 3576.66 [3], which appears also in $a \mathrm{Cyg}$; it is blended with $\mathrm{Fe}$ and $\mathrm{Sc}^{+}$lines.

$Y$. The strongest line is 3620.97 [400]; it may contribute to 3621.08 of a Cyg, which is due mainly to $\mathrm{Fe} 3621.47$.

TABLE VIII

\begin{tabular}{c|c|c}
\hline \hline Lab. $\lambda$ & Lab. Int. & $\odot$ Int. \\
\hline $3832.45 \ldots \ldots \ldots \ldots$ & 100 & $3 \mathrm{~N}$ \\
$3788.70 \ldots \ldots \cdots \cdots$ & 200 & 2 \\
$3774.37 \ldots \ldots \ldots \cdots$ & 300 & 3 \\
$3709.77 \ldots \ldots \ldots \cdots$ & 500 & 3 \\
\hline
\end{tabular}

$Y^{+}$. The lines shown in Table VIII contribute to blends.

$Z r$. The coincidences observed seem due to chance.

$\mathrm{Zr}^{+}$. The two strongest lines, 3698.17 and $375^{\mathrm{I}} .59$, are blended with $H_{0}$ and $H_{\kappa}$; certain other $Z r^{+}$lines may possibly contribute faintly to blends.

$B a^{+}$. The region is not suitable; it seems improbable that the line at 389 I. 78 [50] could appreciably blend with $\mathrm{Fe}$ 91.94.

$\mathrm{La}^{+}$. The strongest line is located at 3794.77 [500]; it may possibly blend slightly with $\mathrm{Fe}$ 3795.or. Other coincidences seem accidental.

$\mathrm{Ce}^{+}$. Higher dispersion is needed; $380 \mathrm{r} .53$ [10] may possibly contribute to $380 \mathrm{r} .53$ in $a \mathrm{CVn}$.

$E u^{+}$. The presence of $E u^{+}$in $a \mathrm{CVn}$ is confirmed.

Rare earths. There are many coincidences with lines of ionized $\operatorname{Pr}, N d, S m, G d, T b, D y$, and $E r$, but higher dispersion is required for determining with certainty the reality of the identifications.

PECULIAR A STARS

A number of lines are present in the peculiar stars only; for example, twenty-one lines are present in a CVn but not in the ordinary 
Ao stars; there are four similar lines in a And, eight in $\theta$ Aur, and nine in $\epsilon$ UMa. Many of these lines coincide in position with $F e$ lines, but it is certain that in many cases this identification is not satisfactory.

We wish to express our thanks to Professor Otto Struve, who suggested and encouraged this investigation; to Dr. F. E. Roach, who obtained the spectrograms; and to Dr. W. W. Morgan, who gave valuable advice.

September II, I935 